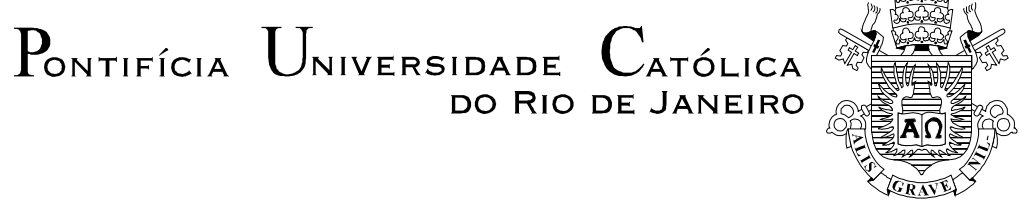

Victor Andres Ayma Quirita

\title{
Avaliação de Métodos de Otimização Aplicados no Modelamento da Estrutura dos Vasos Sanguíneos
}

\begin{abstract}
Dissertação de Mestrado
Dissertação apresentada como requisito parcial para obtenção do título de Mestre pelo Programa de PósGraduação em Engenharia Elétrica da PUC-Rio.
\end{abstract}

Orientador: Prof. Raul Queiroz Feitosa Co-Orientador: Dr. Dário Augusto Borges Oliveira

Rio de Janeiro

Julho de 2013 


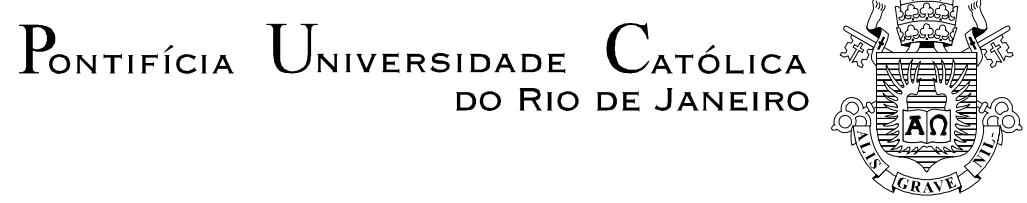

Victor Andres Ayma Quirita

\section{Avaliação de Métodos de Otimização Aplicados no Modelamento da Estrutura dos Vasos Sanguíneos}

Dissertação apresentada como requisito parcial para obtenção do título de Mestre pelo Programa de Pós-Graduação em Engenharia Elétrica da PUC-Rio. Aprovada pela Comissão Examinadora abaixo assinada.

Prof. Raul Queiroz Feitosa Orientador Pontifícia Universidade Católica do Rio de Janeiro

Dr. Dário Augusto Borges Oliveira Co-Orientador

Pontifícia Universidade Católica do Rio de Janeiro

Prof. Juan Guillermo Lazo Lazo Pontifícia Universidade Católica do Rio de Janeiro

Prof. Maria Luiza Fernandes Velloso Universidade do Estado do Rio de Janeiro

Prof. Gilson Alexandre Ostwald Pedro da Costa Pontifícia Universidade Católica do Rio de Janeiro

Prof. José Eugenio Leal Coordenador Setorial do Centro Técnico Científico - PUC-Rio 
Todos os direitos reservados. É proibida a reprodução total ou parcial do trabalho sem autorização da universidade, do autor e do orientador.

\section{Victor Andres Ayma Quirita}

Nasceu em Cusco, Peru, no ano 1986. No 2008 se gradou na Universidad Nacional San Antonio Abad del Cusco na especialidade de Engenharia Eletrônica com ênfases em Telecomunicações. Atualmente esta fazendo o mestrado no programa de Engenharia Elétrica da PUC-Rio na área de Processamento de Sinais e Controle. Suas principais áreas de interesse são o processamento digital de imagens, visão computacional e telecomunicações.

Ficha Catalográfica

Ayma Quirita, Victor Andres
Avaliação de métodos de otimização aplicados no
modelamento da estrutura dos vasos sanguíneos / Victor
Andres Ayma Quirita; orientador: Raul Queiroz Feitosa ;
co-orientador: Dário Augusto Borges Oliveira. - 2013.
78 f. : il. (color.) ; $30 \mathrm{~cm}$
Dissertação (mestrado)-Pontifícia Universidade
Católica do Rio de Janeiro, Departamento de Engenharia
Elétrica, 2013.
Inclui bibliografia
1. Engenharia elétrica - Teses. 2. Vasos sanguíneos.
3. Imagens médicas. 4. Segmentação. 5. Métodos de
otimização. 6. Tomografia computadorizada. I. Feitosa,
Raul Queiroz. II. Oliveira, Dário Augusto Borges. III.
Pontifícia Universidade Católica do Rio de Janeiro.
Departamento de Engenharia Elétrica. IV. Título.

CDD: 621.3 
A Deus, por me permitir despertar cada dia e olhar sua grandeza.

A meus pais, Victor e Rina, pelo seu exemplo, apoio e amor incondicional.

A meus irmãos, Hugo, Paola e Isarina, por estar sempre para mim.

A Maybee, amiga, companheira, amor de minha vida, teu coração foi a motivação de todos meus dias. 


\section{Agradecimentos}

Ao meu orientador Raul Queiroz Feitosa, pela oportunidade, compreensão, apoio, motivação, orientação e amizade, dentro e fora do âmbito acadêmico.

A Dário Oliveira, por todo seu apoio, tempo, colaboração, orientação, paciência e amizade. Sem sua ajuda e comentários não poderia ter sido realizada esta pesquisa.

À PUC-Rio e aos seus professores do Departamento de Engenharia Elétrica.

Ao CNPq pelos auxílios concedidos.

A meu irmão Hugo, por seus comentários, ideias e observações que me ajudaram a realizar grande parte deste trabalho.

Aos meus amigos e colegas da PUC-Rio, pela amizade, apoio e alegrias. 


\section{Resumo}

Quirita, Victor Andres Ayma; Feitosa, Raul Queiroz (Orientador); Oliveira, Dário Augusto Borges (Co-Orientador). Avaliação de Métodos de Otimização Aplicados no Modelamento da Estrutura dos Vasos Sanguíneos. Rio de Janeiro, 2013. 78p. Dissertação de Mestrado Departamento de Engenharia Elétrica, Pontifícia Universidade Católica do Rio de Janeiro.

Segundo relatórios da Organização Mundial da Saúde, as doenças cardiovasculares são a principal causa de óbitos em nível mundial. Podem, porém, ser controladas mediante diagnóstico e tratamento adequados. Nesse contexto, as ferramentas tecnológicas de auxílio ao diagnóstico são importantes para redução do número de óbitos causados por este tipo de doenças. Esta dissertação avalia aos métodos de otimização: Differential Evolution, Generalized Pattern Search, Mesh Adaptive Direct Search e Nelder-Mead Algorithm, aplicados na busca dos parâmetros que modelam a estrutura dos vasos sanguíneos a fim de melhorar os resultados e tempo de processamento da segmentação da árvore vascular em imagens médicas, conforme proposto em (Oliveira, 2013). Neste trabalho, são apresentados conceitos anatômicos e as características das imagens usadas neste estudo. São ainda descritos os métodos de otimização avaliados e a metodologia da segmentação da árvore vascular cujos parâmetros se deseja otimizar. Com essa base, se formula a metodologia de avaliação destes métodos através de uma análise quantitativa, que é produto da formulação de um teste de hipóteses da diferença entre a avaliação média em combinação pareada. Este teste avalia o desempenho dos métodos de otimização quando são aplicados em amostras aleatoriamente escolhidas em cada um dos exames de tomografia computadorizada que pertencem ao banco de dados composto por imagens: sintéticas, coronárias, hepáticas e de fibras nervosas do sistema olfativo. Conforme aos resultados do teste de hipótese, o método de otimização com o melhor desempenho, em acurácia e custo computacional, é escolhido e as conclusões deste trabalho são elaboradas assim como também as propostas de trabalhos futuros nesta mesma linha de pesquisa.

\section{Palavras-chave}

Vasos sanguíneos; Imagens Médicas; Segmentação; Métodos de Otimização; Tomografia Computadorizada. 


\section{Abstract}

Quirita, Victor Andres Ayma; Feitosa, Raul Queiroz (Advisor); Oliveira, Dário Augusto Borges (Co-advisor). Assessment of Optimization Methods applied in Modeling the Structure of Blood Vessels. Rio de Janeiro, 2013. 78p. MSc. Dissertation - Departamento de Engenharia Elétrica, Pontifícia Universidade Católica do Rio de Janeiro.

According to the World Health Organization reports, cardiovascular diseases are the worldwide leading cause of death. However, they can be controlled using proper diagnosis tools and treatments. In this way, the diagnosis assisted technological tools are important to reduce the number of deaths caused by this type of diseases. This dissertation assess optimization methods (Differential Evolution, Generalized Pattern Search, Mesh Adaptive Direct Search, Nelder-Mead Algortihm) applied in the search of parameters that model blood vessels structures in order to improve the results and processing time of a vascular tree segmentation method in medical images, as proposed in (Oliveira, 2013). In this work, anatomical concepts and the characteristics of the images used in this study are presented. The optimization methods assessed and the methodology for the segmentation of the vascular tree, whose parameters are to be optimized, are described. Based on that, the procedure to assess different optimization methods is formulated through a quantitative analysis using a hypothesis test formulation of difference between paired means. This test evaluates the performance of the optimization methods using randomly chosen samples in a computerized tomography exams database composed by synthetic, coronary, hepatic and nervous fiber of the olfactory system images. According to the hypothesis test results, the optimization method with the best performance, both in accuracy and computational cost, is selected and the conclusions of this work are elaborated as well as the proposals for further research.

\section{Keywords}

Blood vessels; Medical Images; Segmentation; Optimization Methods; Computerized Tomography. 


\section{Sumário}

1 Introdução 15

1.1. Motivação 17

1.2. Objetivos 17

1.3. Estrutura da Dissertação 18

2 Vasos Sanguíneos e sua Representação em Imagens Médicas de $\begin{array}{ll}\text { Tomografia Computadorizada } & 19\end{array}$

2.1. Vasos Sanguíneos 19

2.2. Tomografia Computadorizada (CT) 21

2.3. Imagens Digitais 24

2.4. Padrão DICOM 24

3 Segmentação da Árvore Vascular 26

3.1. Introdução 26

3.2. Modelo da Amostragem Cônica 27

3.3. Detecção de Ponto do Vaso Sanguíneo 28

3.3.1. Cálculo da Vascularidade $\quad 29$

3.3.2. Seleção do Ponto Candidato de Vaso 31

3.4. Rastreamento da Rede Vascular 32

3.4.1. Detecção da Rede Vascular 32

3.4.2. Validação da Rede Vascular 33

3.5. Definição das Novas Sementes 34

3.6. Contextualização do Trabalho 34

4 Fundamentos Teóricos $\quad 35$

4.1. Otimização Matemática 35

4.1.1. Problema de Otimização Matemática 35

4.1.2. Tipos de Problemas de Otimização 36

4.1.3. Otimalidade $\quad 37$

4.2. Evolução Diferencial 38

4.2.1. Descrição do Método 39

4.2.2. Mutação 39 
4.2.3. Cruzamento 40

4.2.4. Seleção 41

4.3. Busca Generalizada de Padrões $\quad 41$

4.3.1. Fundamentos e Terminologia 42

4.3.2. Etapa SEARCH 45

4.3.3. Etapa POLL 46

4.4. Busca Direta de Malha Adaptativa $\quad 47$

4.4.1. Etapa POLL no MADS 48

4.5. Algoritmo Nelder - Mead 51

4.5.1. Inicio do Simplex 52

4.5.2. Reflexão do Simplex 53

4.5.3. Expansão do Simplex 53

4.5.4. Contração do Simplex 54

4.5.5. Redução do Simplex 54

5 Metodologia para a Aplicação dos Métodos de Otimização 56

5.1. Função Objetivo 56

5.2. Implementação dos Métodos de Otimização 57

5.3. Principais Parâmetros do Funcionamento dos Protótipos 58

5.4. Banco de Imagens 59

5.5. Procedimento Experimental 62

6 Avaliação Experimental $\quad 65$

6.1. Avaliação Quantitativa 65

6.2. Avaliação Visual 70

7 Conclusões $\quad 73$

7.1. Discussão 73

7.2. Trabalhos Futuros $\quad 74$

7.3. Considerações Finais $\quad 74$

$\begin{array}{ll}\text { Referências Bibliográficas } & 76\end{array}$ 


\section{Lista de figuras}

Figura 1. Sistema Cardiovascular. 20

Figura 2. Estrutura comparativa dos vasos sanguíneos (Tortora, 2007). 21

Figura 3. Exemplo de uma imagem de CT da região abdominal (de esquerda para direita, e de cima para baixo: vistas axial, coronal e sagital). 22

Figura 4. Representação dos vasos sanguíneos em imagens de CT. 23

Figura 5. CT exame representado como uma imagem 3D. 25

Figura 6. Grafo construído usando as direções locais da rede vascular (Oliveira, 2013). 26

Figura 7. Diagrama de fluxo do método para segmentar as redes vasculares (Oliveira, 2013). 27

Figura 8. Modelo de amostragem cônico (Oliveira, 2013). 28

Figura 9. Etapas do modelo para o cálculo da vascularidade (Oliveira, 2013). 29

Figura 10. Seleção dos pontos amostrados (Oliveira, 2013). 32

Figura 11. Rastreamento de redes vasculares no grafo com a detecção dos vasos nos seus nós (Oliveira, 2013). 32

Figura 12. Regras do post-processamento estabelecem algumas restrições anatômicas para a rede vascular (Oliveira, 2013). 33

Figura 13. Definição de novas sementes (Oliveira, 2013). 34

Figura 14. Mínimo Global e Local de $f(x) \quad 37$

Figura 15. Linhas de contorno de uma função objetivo bidimensional, e o processo de mutação para gerar $v_{i, G+1}$, (Storn \& Price, 1997). 40

Figura 16. Processo de cruzamento para $n=7$ parâmetros, (Storn \& Price, 1997).

Figura 17. Pontos de prova ao redor de $x_{k}$ (Abramsom, et al., 2003). 43

Figura 18. Estrutura geral do método GPS (MathWorks, 2012). 44

Figura 19. Pontos gerados no algoritmo UD para o caso bidimensional. $\quad 46$

Figura 20. GPS: Exemplo de pontos de prova $P_{k}=\left\{x_{k} \pm \Delta_{k}^{m} e j:\right.$ ej $\left.\in E\right\}=$ $\{p 1, p 2, p 3\}$ para diferentes valores de $\Delta_{k}^{m}=\Delta_{k}^{p}$ (Audet \& Dennis, 2006). 
Figura 21. MADS: Exemplo de pontos de prova $P_{k}=\left\{x_{k} \pm \Delta_{k}^{m} d j: d j \in D\right\}=$ $\{p 1, p 2, p 3\}$ para diferentes valores de $\Delta_{k}^{m}$ e $\Delta_{k}^{p}$ (Audet \& Dennis, 2006).

Figura 22. Descrição do algoritmo Nelder - Mead (Luersen, et al., 2004). 51

Figura 23. Processo de reflexão para $\operatorname{Pr}$ (Mathews \& Fink, 2004). 53

Figura 24. Processo de expansão para $P e$ (Mathews \& Fink, 2004). 53

Figura 25. Processo de contração para $P c$ (Mathews \& Fink, 2004). 54

Figura 26. Processo de redução em direção de $P l$ (Mathews \& Fink, 2004). 54

Figura 27. (a) Vista sagital de um vaso no exame Synthetic1, (b) Representação aproximada do seu modelo 3D.

Figura 28. (a) Vista sagital de um vaso no exame Synthetic2, (b) Representação aproximada do seu modelo 3D.

Figura 29. (a) Vista coronal de um vaso no exame Synthetic3, (b) Representação aproximada do seu modelo 3D.

Figura 30. (a) Vista axial de um vaso no exame OPF1, (b) Representação aproximada do seu modelo 3D.

Figura 31. (a) Vista axial de um vaso no exame OPF3, (b) Representação aproximada do seu modelo 3D.

Figura 32. (a) Vista axial de um vaso no exame OPF7, (b) Representação aproximada do seu modelo 3D.

Figura 33. Em vermelho, escolha aleatória de alguns dos 50 pontos identificados como pertencentes ao vaso no exame de CT.

Figura 34. Aplicação do método $D E$ (esquerda) e $N M$ (direita) no exame Synthetic1.

Figura 35. Aplicação do método $D E$ (esquerda) e $N M$ (direita) no exame Synthetic2.

Figura 36. Aplicação do método $D E$ (esquerda) e $N M$ (direita) no exame Synthetic3.

Figura 37. Aplicação do método $D E$ (esquerda) e $N M$ (direita) no exame OPF1.

Figura 38. Aplicação do método $D E$ (esquerda) e $N M$ (direita) no exame OPF3.

Figura 39. Aplicação do método $D E$ (esquerda) e $N M$ (direita) no exame OPF7. 


\section{Lista de tabelas}

Tabela 1. Decisões lógicas para o algoritmo NM (Mathews \& Fink, 2004). 52

Tabela 2. Características dos métodos de otimização implementados. $\quad 57$

Tabela 3. Parâmetros de configuração dos algoritmos de otimização. 58

Tabela 4. Banco de Imagens de CT preparado na pesquisa. 59

Tabela 5. Parte da base de dados com a vascularidade de 20 pontos na imagem Synthetic1. 63

Tabela 6. Valores do Pvalue para o teste de hipótese. 66

$\begin{array}{ll}\text { Tabela 7. Valor dos CI nos testes de hipótese realizados. } & 67\end{array}$

Tabela 8. Número de execuções dos métodos estudados. 69 


\title{
Lista de Símbolos e Abreviações
}

\author{
2D Bidimensional \\ 3D Tridimensional \\ CT Computed Tomography \\ DE Differential Evolution \\ DICOM Digital Imaging Communications in Medicine \\ DS Direct Search \\ GPS Generalized Pattern Search \\ MADS Mesh Adaptive Direct Search \\ NM Nelder - Mead \\ OMS Organização Mundial da Saúde \\ OPS Organização Pan-Americana da Saúde \\ WHO World Health Organization \\ UD Uniform Distribution
}


O Senhor é o meu pastor e nada me faltará. Deitame em verdes pastos e guia-me mansamente em águas tranqüilas. Refrigera a minha alma, guia-me pelas veredas da justiça, por amor do seu nome. Ainda que eu ande pelo vale da sombra da morte, não temerei mal algum, porque Tu estás comigo; a Tua vara e o Teu cajado me consolam. Prepara-me uma mesa perante os meus inimigos, unges a minha cabeça com óleo, o meu cálice transborda. Certamente que a bondade e a misericórdia me seguirão todos os dias da minha vida e habitarei na casa do SENHOR por longos dias.

Bíblia, Salmos, 23 (Salmo de Davi). 